\title{
COR PULMONALE IN COAL-WORKER'S PNEUMOCONIOSIS
}

\author{
BY \\ A. L. WELLS* \\ From the Institute of Pathology, Welsh National School of Medicine, Cardiff \\ Received September 26, 1953
}

Attention has been drawn to the occurrence of pulmonary heart disease in South Wales coalworkers suffering from severe pneumoconiosis. The clinical, radiological, and electrocardiographic features have been described by Thomas (1948) and, assessing right ventricular hypertrophy post mortem by a technique of differential ventricular weighing, he concluded that cardiographic patterns are poor indicators of early right ventricular hypertrophy (1951). Gooding (1946) distinguished between two types of cardiac failure encountered post mortem in Welsh anthracite miners- $-(a)$ congestive failure with peripheral odema and ascites, and $(b)$ terminal failure with pulmonary odema. Right ventricular hypertrophy and dilatation were constant in almost all the cases that terminated in one of those ways, and the signs of right heart strain were evident in the advanced stages, as well as in those dying from cardiac failure.

Gough (1940 and 1946) pointed out that hypertrophy of the right heart is common in massive fibrosis, that death may result from congestive cardiac failure, and that pulmonary thrombosis often seemed to have been the cause of death.

In the U.S.A., numbers of similar reports have appeared dealing with the pathology of the cardiovascular complications of industrial lung fibrosis (e.g. Jaffé, 1934; Dyson, 1934; Coggin, et al., 1938; and Geever, 1947). These have amply confirmed the occurrence of right heart failure in such cases, but have often included classical silicosis with coal-worker's pneumoconiosis or have failed to specify the particular industrial process. Gardner (1940) thought that in death from silicosis the heart was generally already damaged by arteriosclerosis or other causes, and was sceptical of an autopsy finding of right heart hypertrophy without arteriosclerosis is such cases. However, he conceded that areas of conglomerate fibrosis and emphysema in the lungs might add to a cardiac burden already imposed for some other reason.

This paper is concerned with an analysis of the autopsy findings in 388 Welsh coal-workers, $\dagger$ with a view to establishing the frequency with which cor pulmonale heart failure occurred due solely to the industrial lung disease encountered. In another paper (Wells, in press) the anatomical changes in the lung vessels have been described. Some degree of change was always seen, though it was usually slight in simple pneumoconiosis. Severe obliterative and destructive changes in the pulmonary vessels, large and small, were seen in the advanced " massive fibrotic" stage of the disease, whether accompanied by recognizable evidence of tuberculosis or not. This was accompanied by variable degrees of enlargement of the bronchial arteries, which was thought to be an attempt to compensate for the rising pulmonary arterial pressure that resulted from loss of the pulmonary vessels. Thrombotic episodes were frequent in the pulmonary arteries, especially in the areas involved in massive fibrosis.

Cardiac failure was a common cause of death, and as might be expected when the average age

* Late pathologist to M.R.C. Pneumoconiosis Research Unit, South Wales; now at Institute of Pathology, Belfast.

$\dagger$ An account of the first 243 cases in this series was presented as a thesis for the degree of Doctor of Medicine of the Queen's University of Belfast. 
at death was 61 years, was frequently due, at least in part, to systemic hypertension, coronary arterial disease, and valvular lesions. When these causes were excluded, however, nearly half the men who suffered from the advanced form of coal-worker's pneumoconiosis (progressive massive fibrosis) had died from right-sided heart failure that could be ascribed entirely to their industrial lung disease. With early simple pneumoconiosis, cor pulmonale heart failure occurred frequently, but could rarely be attributed to the industrial disease.

\section{Material AND Methods}

All the 388 cases were underground colliery workers from the Rhondda, Merthyr, and Aberdare valleys, except two coal-trimmers handling the same type of semi-bituminous (steam) coal. These men came to autopsy in the Institute of Pathology of the Welsh National School of Medicine. Unfortunately they do not as a whole constitute a random sample of the coal-mining population in this coal-field, because there was a heavy bias favouring those whose relatives believed there were grounds for compensation, as well as a high proportion of proven cases of pneumoconiosis. All degrees of severity of the disease process were observed, however, because of the wide age range of subjects.

The autopsies were all performed in the usual way by the writer, excepting that one lung was preserved for subsequent sectioning by the Gough and Wentworth technique; the other lung was dissected according to the German technique redescribed by Belt (1936), by which method the vessels are exposed down to their finest branches. In cases with massive dust fibrosis, a portion of the fibrotic area about $2 \mathrm{~cm}$. in diameter was taken, if possible from an area showing suspicion of tuberculosis, for guinea-pig inoculation. In the heart, in addition to routine weighing and dissection, the thicknesses of the muscular parts of the right and left ventricles were measured on their anterior walls. A thickness of $5 \mathrm{~mm}$. was accepted as the upper limit of normal for the right ventricle. In equivocal cases, account was taken of the general anatomical features of the cavity as a whole. The general pathological anatomy of the disease has been already fully described, (Gough, 1940, 1944, 1946; Fletcher and Gough, 1950; and Heppleston, 1947), and will only be referred to as required.

The 388 cases are to be considered in three groups-simple pneumoconiosis, 181 cases; massive pneumoconiosis, 136 cases; and tuberculosis, 71 cases. Cases were put into the tuberculous category irrespective of the presence or degree of pneumoconiosis if they showed conventional macroscopic or microscopic evidence of tuberculosis of any organ, or if the guinea-pig inoculation proved positive for tuberculosis. The distinction between simple and massive disease was usually easy; examination of the Gough and Wentworth section was of great assistance in a few borderline cases.

\section{Simple PNEUmoconiosis}

The 181 cases of simple pneumoconiosis were subdivided with a view to identifying those that could be considered to have shown and died from cor pulmonale heart failure (Table 1). A total of 110 died primarily from acute or chronic cardiac failure, and 71 from other causes.

From the 110 cardiac cases 47 were excluded either because they had sustained coronary occlusion recognizable by the findings of coronary thrombosis and/or myocardial infarction, or because they showed severe syphilitic coronary ostial narrowing (3 cases). Those with slight coronary atheroma were not excluded, as according to Harrison and Wood (1949), even moderate degrees of atheroma do not necessarily cause narrowing during life. A further 25 considered to have died from systemic hypertensive cardiac failure and 9 with valvular disease of the heart, were examined separately. Those with both systemic hypertension and significant coronary atheroma were grouped according to the major lesion presenting at autopsy.

The degree of simple pneumoconiosis in these 81 cases that died of heart failure other than that due to cor pulmonale will be considered briefly. This is probably given best by estimating the presence and severity of focal emphysema. They included only 7 with a fairly severe degree of focal emphysema and another 4 with any significant degree of general emphysema. The majority of them showed only moderate degrees of dust pigmentation, and there seem no grounds for believing that any special tendency towards coronary disease or hypertension existed in these miners by 
TABLE I

CAuses of Death

\begin{tabular}{l|c|c}
\hline & $\begin{array}{c}\text { Simple } \\
\text { pneumoconiosis }\end{array}$ & $\begin{array}{c}\text { Massive } \\
\text { pneumoconiosis }\end{array}$ \\
\hline Coronary occlusion & 47 & 18 \\
Systemic hypertension & 25 & 14 \\
Cardiovascular disease & 9 & 5 \\
Adrenaline poisoning & 1 & 0 \\
Cor pulmonale $\begin{array}{l}\text { miscellaneous } \\
\text { pneumoconiosis }\end{array}$ & 24 & 0 \\
\hline Cardiac failure & 110 & 57 \\
Other causes & 71 & 94 \\
\hline Total & 181 & 42 \\
\hline
\end{tabular}

virtue of the state of their lungs. Only three showed further lung changes, one having bronchiectasis, one pulmonary thrombosis and infarction, and one (not included above) asthma; the last died from an overdose of adrenaline.

The remaining 28 cases died from right-sided cardiac failure. Although they all had simple pneumoconiosis, most of them also had other pulmonary lesions, which after exclusion left only 4 with cor pulmonale due to simple pneumoconiosis alone. In view of the apparently slight anatomical interference with the pulmonary vasculature this is not surprising. They all showed right ventricular hypertrophy. The most common complicating lesions in this group were general emphysema and bronchiectasis, and usually the slight degree of simple pneumoconiosis present left no doubt that it was not mainly responsible for the cardiac failure.

The four cases with cor pulmonale due to simple pneumoconiosis included three on the border line between simple and massive pneumoconiosis, with small infective nodules and well marked emphysema in the vicinity of these lesions: there was as well a good deal of undoubted focal emphysema elsewhere. The fourth showed only the lesions of simple disease with severe focal emphysema, and he was the only one in which it could be said with confidence that he was free of general emphysema.

\section{Massive PNeumoconiosis}

Ninety-four of the 136 cases died with evidence of congestive cardiac failure (Table I), some with causes other than pneumoconiosis. In such mixed cases the severe degree of pneumoconiosis present contributed something towards the development of cardiac failure; they were excluded in order to assess the frequency with which massive pneumoconiosis alone brought about death in cardiac failure. The complicated cases are dealt with first. There were 5 with valvular disease of the heart, 3 with calcific aortic disease, 1 with mitral calcification, and 1 with both mitral and aortic lesions; progressive massive fibrosis was severe in only 2 of them. There were 14 cases of systemic hypertension; their lungs showed all stages of progressive massive fibrosis and, in general, the emphysema encountered did not seem severe. Eighteen cases of coronary disease were included, 15 with myocardial infarction and/or thrombotic occlusion of a coronary branch; again, the severity of the lung lesion was variable and bore no apparent relation to the cardiac changes. Out of the remaining 99 cases, 42 died from miscellaneous causes, leaving 57 cases of cor pulmonale.

Massive Pneumoconiosis with Cor Pulmonale. Cases of systemic hypertension and valvular disease were excluded, and considerations regarding cases with coronary atheroma were the same as in the simple pneumoconiosis group. One case had hitherto unsuspected early syphilitic aortitis unassociated with valvular or coronary arterial lesions. Their ages were from 38 to 79 years, averaging $59 \cdot 8$ years (56 cases). Fifty-four had heart weights from $200 \mathrm{~g}$. to $530 \mathrm{~g}$., averaging $375 \mathrm{~g}$. Right ventricular thicknesses were increased in all except three, measuring $4 \mathrm{~mm}$., $5 \mathrm{~mm}$., and $5 \mathrm{~mm}$. 
respectively, the first of whom had acute cor pulmonale from massive pulmonary embolism. The thicknesses ranged from $4 \mathrm{~mm}$. to $9 \mathrm{~mm}$. and averaged $6.9 \mathrm{~mm}$.

It is possible to gain further confirmation of the cardiac hypertrophy, by comparing the simple ratio, heart weight/body weight, for 54 cases for which figures were available with those for 96 men who were not miners with normal hearts and lungs, obtained from the post-mortem records of this department. The ratios of 76 per cent of the coal-workers with cor pulmonale and massive fibrosis were greater than 0.007 , while those of 87 per cent of the control group were less than this.

Fifteen of these cases showed thrombosis of a major branch of the pulmonary artery, usually that to one or other upper lobe which was the site of massive fibrosis. One showed evidence of pulmonary embolism as well, and a further one, already referred to, had massive pulmonary embolism resulting in acute cor pulmonale and death. Little comment is required about the actual degrees of pneumoconiosis encountered. All had well established massive fibrosis, nearly always associated with bullous emphysema which was usually severe. No other pulmonary lesions were found except terminal bronchopneumonia and pulmonary thrombosis.

Massive Pneumoconiosis with Death not due to Heart Failure. None of the remaining 42 cases showed evidence of having suffered from congestive cardiac failure. Some of them showed evidence of right ventricular strain in hypertrophy of that chamber, after causes other than pneumoconiosis were excluded. Thus, of 30 cases with no other lung lesion except massive fibrosis and with no other cardiovascular abnormalities, 10 had right ventricles more than $5 \mathrm{~mm}$. thick. These 16 cases did not appear to have less severe dust disease as a group. They may have acquired their massive lesions much later in life, and thus not had time to reach the threshold of vascular interference at which cardiovascular effects become manifest.

\section{TUBERCULOSIS}

The 71 tuberculous cases from a somewhat heterogenous group, from which it is difficult to draw any conclusions as to the cardiovascular changes, because so many deaths were due to direct and indirect effects of the tuberculosis itself (when it was present in an active form) and also to other unrelated causes. Thus out of 60 cases with extensive chronic lung lesions, 29 died of tuberculous lesions such as miliary tuberculosis, tuberculous bronchopneumonia, enteritis, or meningitis, singly or in combinations, associated in one with extensive amyloid disease. Twelve of these had right ventricular hypertrophy, but showed no signs of being in a state of cardiac failure.

Of the remaining 31 cases, 19 died from miscellaneous causes, including coronary occlusion and congestive cardiac failure: 12 are left and can be considered as having died from cor pulmonale heart failure. Eight had bilateral massive fibrotic lesions showing evidence of tuberculous activity. One other showed evidence of slight miliary spread. Another showed massive fibrosis which yeilded a positive guinea-pig reaction. There were single cases of bilateral massive fibrosis with unilateral cavitating tuberculosis, and of bilateral massive fibrosis (itself not manifestly tuberculous) associated with recognizable diaphragmatic and pleural tuberculosis.

\section{Discussion}

In cases of simple disease death could not often be attributed to cor pulmonale heart failure from industrial disease alone. They were cases in which it was difficult to know if the correct classification, was "simple" or " massive." All showed well marked emphysema, which was considered to be predominantly focal.

In view of the advanced age at which death generally occurred, the lethal nature of massive pneumoconiosis is shown by the fact that 57 out of the 136 cases $(42 \%)$ had died directly from the cardiovascular effects of their severe lung disease. Moreover, in the others with cardiac failure, particularly those with systemic hypertension and valvular lesions, massive fibrosis and emphysema must have contributed materially to the cardiac burden. Whether the consequences of coronary occlusion and myocardial infarction are influenced to any extent by severe pneumoconiosis is a 
difficult question, but in some cases there must be a deleterious effect. Probably when coronary arterial disease is the main cause of a fairly prolonged cardiac failure, massive fibrosis, when present, is a materially contributing factor. It is, however, difficult to believe that sudden death precipitated by " acute coronary insufficiency" is affected in any way by the state of the lungs.

In those with tuberculous complications, a full evaluation of the cardiovascular changes is often precluded by the presence of a fatal form of tuberculosis, such as meningitis or bronchopneumonia.

However, this is not invariable and cases were seen who had cor pulmonale and died from that form of heart failure. The usual clinical picture is predominantly that of the respiratory disability of pulmonary tuberculosis and its sequelæ, but cardiovascular effects due to dust fibrosis sometimes co-exist with active tuberculosis.

\section{SUMMARY AND CONCLUSIONS}

Necropsies on 388 coal-workers from the Rhondda and neighbouring valleys of the South Wales coal-field were classified into three groups, simple pneumoconiosis, massive pneumoconiosis, and tuberculosis.

Simple pneumoconiosis rarely gave rise to right heart failure by itself, although the two were frequently associated in the presence of other pulmonary disease. In these mixed cases it is doubtful whether the simple pneumoconiosis played a significant part in causing death.

Massive pneumoconiosis (42\%) often gave rise to right heart failure without another cardiac disease. A further 27 per cent of this group died from heart failure, partly due to other lesions such as severe coronary arterial disease, cardiac valvular disease, and systemic hypertension, but the pneumoconiosis probably contributed materially to the cause of death.

The assessment of cases with tuberculosis was complicated by the co-existence of lethal tuberculous lesions with heart failure, but 17 per cent of this group were considered to have died from cor pulmonale heart failure.

I am indebted to Professor Jethro Gough for much help in the preparation and presentation of this work, and to the technical and photographic departments of the Institute of Pathology in the Welsh National School of Medicine, Cardiff.

Belt, T. H. (1936). J. tech. Meth., 15, 39.

\section{REFERENCES}

Coggin, C. B., Griggs, D. E., and Stilson, W. L. (1938). Amer. Heart J., 16, 441.

Dyson, J. M. (1934). Amer. Heart J., 9, 764.

Fletcher, C. M., and Gough, J. (1950). Brit. med. Bull., 7, 42.

Gardner, L. U. (1940). J. Amer. med. Ass., 114, 535.

Geever, E. F. (1947). Amer. J. med. Sci., 214, 292.

Gooding, C. G. (1946). Lancet, 2, 891.

Gordon, H., and Sweets, H. H. Jr. (1936). Amer. J. Path., 12, 545.

Gough, J. (1940). J. Path. Bact., 51, 277.

(1944). Report of Advisory Committee on the Treatment and Rehabilitation of Miners in the Wales Region suffering from Pneumoconiosis. Her Majesty's Stationery Office, London.

- (1946). Lancet, 1, 462.

Harrison, C. V., and Wood, P. (1949). Brit. Heart J., 11, 205.

Heppleston, A. G. (1947). J. Path. Bact., 59, 453.

Jaffé, R. H. (1934). Ill. med. J., 66, 431.

Thomas, A. J. (1948). Brit. Heart J., 10, 282.

— (1951). Brit. Heart J., 13, 1. 